\title{
Performance Characteristics of CdTe Drift Ring Detector
}

\author{
A. Alruhaili, ${ }^{a, b, *}$ P.J. Sellin, ${ }^{b}$ A. Lohstroh, ${ }^{b}$ P. Veeramani, ${ }^{b}$ S. Kazemi, ${ }^{b}$ M. C. Veale, ${ }^{c}$ \\ K. J. S. Sawhney and V. Kachkanov
}

${ }^{a}$ Astronomy Department, King Abdul Aziz University, Jeddah, Saudi Arabia

${ }^{b}$ Physics department, University of Surrey, Guildford, UK

${ }^{c}$ Detector development group, Rutherford Appleton Laboratory, Didcot, UK

${ }^{d}$ Diamond Light Source, Didcot, UK

E-mail: a.alruhaili@surrey.ac.uk, aalruhaili@kau.edu.sa

\begin{abstract}
CdTe and CdZnTe material is an excellent candidate for the fabrication of high energy X-ray spectroscopic detectors due to their good quantum efficiency and room temperature operation. The main material limitation is associated with the poor charge transport properties of holes. The motivation of this work is to investigate the performance characteristics of a detector fabricated with a drift ring geometry that is insensitive to the transport of holes. The performance of a prototype Ohmic CdTe drift ring detector fabricated by Acrorad with 3 drift rings is reported; measurements include room temperature current voltage characteristics (IV) and spectroscopic performance. The data shows that the energy resolution of the detector is limited by leakage current which is a combination of bulk and surface leakage currents. The energy resolution was studied as a function of incident X-ray position with a X-ray microbeam at the Diamond Light Source. Different ring biasing schemes were investigated and the results show that by increasing the lateral field (i.e. the bias gradient across the rings) the active area, evaluated by the detected count rate, increased significantly.
\end{abstract}

KEYWORDS: CdTe; Drift Detector; X-Ray; Synchrotron.

\footnotetext{
* Corresponding author.
} 


\section{Contents}

1. Introduction 1

2. Experimental Method 1

$\begin{array}{ll}2.1 \text { Detector Testing } & 1\end{array}$

2.2 Microbeam Measurements 3

3. Results and Discussion 4

3.1 Spectroscopic Performance $\quad 4$

$\begin{array}{ll}3.2 \text { Microbeam Characterization } & 5\end{array}$

$\begin{array}{ll}\text { 4. Conclusions } & 7\end{array}$

\section{Introduction}

Planar compound semiconductor based detectors are considered an excellent choice for room temperature gamma ray spectroscopy systems due to their good quantum efficiencies in comparison to $\mathrm{Si}$ at high energy[1]. However, the effect of poor hole transport in these materials in some cases represents a critical limitation causing low energy tailing in the spectrum degrading the detector energy resolution [2,3]. Many techniques exist to overcome the problem of poor hole transport, these include, signal processing, novel electrode geometries or a combination of both. Signal processing, such as rise-time-discrimination, where events are rejected that demonstrate a slow component due to hole transport, is associated with rejecting a large number of events reducing detection efficiency [4]. Novel electrode geometries, such as the Co-planar grid technique, are associated with increased electronic noise due to the leakage currents between the grid electrodes [3]. The drift ring technique is an alternative detector geometry that reduces the sensitivity to hole transport, improving the detector energy resolution, without rejecting any events [2,3]. This drift ring technique was first proposed by Gatti and Rehak in 1983 [5] for Si detectors and these are now used extensively for very high resolution X-ray spectroscopy, however, these detectors suffer from poor quantum efficiency at higher energies [5,6]. In 1998, Pamelen and Budtz-Jørgensen [2,3] of the Danish Space Research Institute demonstrated the potential of CdZnTe drift strip detectors for X-ray astronomy applications. In this paper the first results on a prototype CdTe-based drift ring detector are presented.

\section{Experimental Method}

\subsection{Detector Testing}

Figure 1 (Left) shows a cross section of the $1 \mathrm{~mm}$ thick prototype detector studied. The detector was fabricated by Acrorad Ltd with a three ring geometry. Ohmic contacts were chosen rather than Schottky to assure the stability of the detector and to avoid the phenomenon of 
polarization which is associated with time instability under bias [7]. The anode diameter is 0.5 $\mathrm{mm}$ as are the widths of all three rings; individual rings are separated by gaps of $0.5 \mathrm{~mm}$.
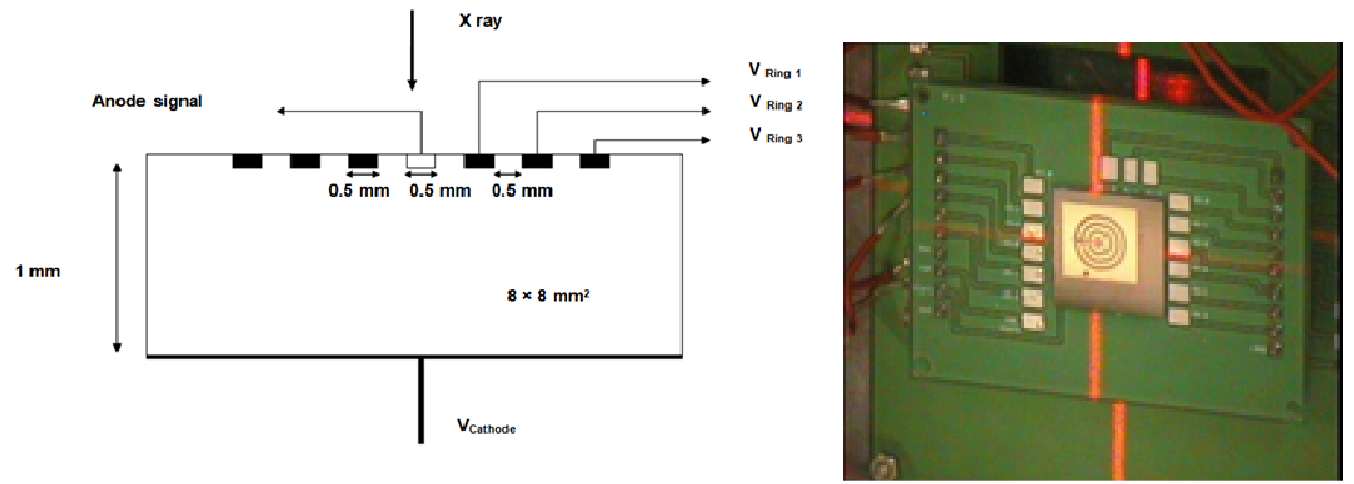

Figure 1 (Left) Cross section of the CdTe drift ring detector and (Right) a photograph of the mounted device at the Diamond Light Source set-up, showing the alignment laser.

The detector was mounted on a PCB (see Figure 1 (Right)) inside an aluminium box. It was connected to an Amptek 250 charge sensitive preamplifier, to a 4 channel power supply (ORTEC 710), a shaping amplifier (ORTEC 570) and a pulse generator (ORTEC 480). Pulse height spectra were acquired using a Canberra Multi-Channel analyser (MCA). The pad electrode and the three rings were negatively biased by connecting them to the 4 channel bias supply and the guard ring was floating. All the pulse height spectra recorded were collected from the central anode.

Initial measurements were made to investigate the effect of the anode leakage current on the spectroscopic performance of the detector. The FWHM of pulser peak height spectra at different electrode bias combinations was used to assess the detector resolution.

The use of different ring biasing schemes allowed the detector performance to be optimized before radiation testing. Under these optimized conditions the spectroscopic performance of the detector was obtained by irradiating the detector with $59.5 \mathrm{keV} \gamma$-rays of a $420 \mathrm{kBq}{ }^{241} \mathrm{Am}$ source. The source was positioned at $50 \mathrm{~mm}$ distance above the centre of the detector and pulse height spectra collected with the MCA. The commercially available Maestro software package was used to display the spectra and analyse the radiation spectra.

An energy calibration of the detector system was made using a charge terminator of capacitance $2.31 \mathrm{pF}$. The shaping amplifier was set to 200 coarse gain, 0.9 fine gain and $3 \mu \mathrm{s}$ shaping time. By connecting a voltage step from a pulse generator to the test input of the Amptek CoolFet preamplifier, pulse height spectra were produced at a number of different MCA channel numbers. Using an oscilloscope, the pulse amplitude of each signal was calculated and the centroid channel number correlated to the induced charge using:

$$
E(k e V)=V(m V) \times C(p F) \times W(e V / e h p) \times 6.25[8]
$$

Eq. 1

Where $\mathrm{C}$ is the capacitance of the charge terminator, $\mathrm{W}$ is the average energy to create one electron-hole pair, equal to $4.4 \mathrm{eV} / \mathrm{ehp}$ in the case of CdTe [9], and 6.25 is a conversion factor. Equation (1) describes when the charge, which was created by the $\mathrm{X}-$ ray, $\mathrm{E} \mathrm{X} \mathrm{/} \mathrm{W} \times 1.6 \times 10$ $19 \mathrm{C}$, and the charge which was created by pulser, $\mathrm{Q}=\mathrm{C} \times \mathrm{V}$, appears in the same channel number of the MCA. 


\subsection{Microbeam Measurements}

Microbeam characterisation of the drift ring detector was carried out on the beam line B16 at the Diamond Light Source Synchrotron [10]. The storage ring was operated at $3 \mathrm{GeV}$ with a current of $250 \mathrm{~mA}$. The X-ray energy was tuned to $25 \mathrm{keV}$ by a Si $\left(\begin{array}{lll}1 & 1 & 1\end{array}\right)$ double crystal monochromator; a small amount of flux is also produced at the 3rd harmonic which has an energy of $75 \mathrm{keV}$. The beam spot size was collimated to $20 \mu \mathrm{m} \times 20 \mu \mathrm{m}$ using a set of tungsten slits and scanned across the detector in a number of line scans. A $7.5 \mathrm{~mm}$ thick aluminium (Al) absorber was used to reduce the beam intensity to $\sim 1 \times 10^{9}$ photons $\mathrm{s}^{-1} \mathrm{~cm}^{-2}$; this hardened the $\mathrm{X}$-ray beam changing the ratio of the first harmonic $(25 \mathrm{keV})$ and third harmonic $(75 \mathrm{keV}) \mathrm{X}$ ray lines. Table 1 summarises the beam specifications which were used for the line scan measurements.

\begin{tabular}{|l|l|}
\hline X-Ray Beam Size & $20 \mu \mathrm{m}$ x $20 \mu \mathrm{m}$ \\
\hline X/Y Step Size & $100 \mu \mathrm{m}$ \\
\hline Acquisition Time Per Position & $120 \mathrm{~s}$ \\
\hline $1^{\text {st }}$ Harmonic Energy & $25 \mathrm{keV}$ \\
\hline $3^{\text {rd }}$ Harmonic Energy & $75 \mathrm{keV}$ \\
\hline
\end{tabular}

Table 1: The specification of the X-ray microbeam measurements.

A coarse positioning to the central anode of the detector was made using a computer controlled X/Y stage and a laser beam which had been pre-aligned to the X-ray beam. A 2D scan was conducted from this as the initial starting point in $0.1 \mathrm{~mm}$ steps. A $60 \mathrm{~s}$ acquisition was made at each position with the pad electrode voltage at $-100 \mathrm{~V}$ and the ring voltages to $20 \mathrm{~V},-40 \mathrm{~V}$ and $-60 \mathrm{~V}$ for the $1 \mathrm{st}, 2 \mathrm{nd}$ and $3 \mathrm{rd}$ ring respectively. The $2 \mathrm{D}$ scan showed a plateau of 25 and $75 \mathrm{keV}$ peak count rates which drops significantly when the scan position exceeded the anode area. Based on these measurements, the centre of the anode was determined to be the middle of the plateau within $\pm 100 \mu \mathrm{m}$. Once the beam position relative to the anode was aligned line scan measurements were made for a number of different bias schemes. Since the drift ring geometry of the detector has rotational symmetry, line scans were only made in the + $Y$ vertical direction.

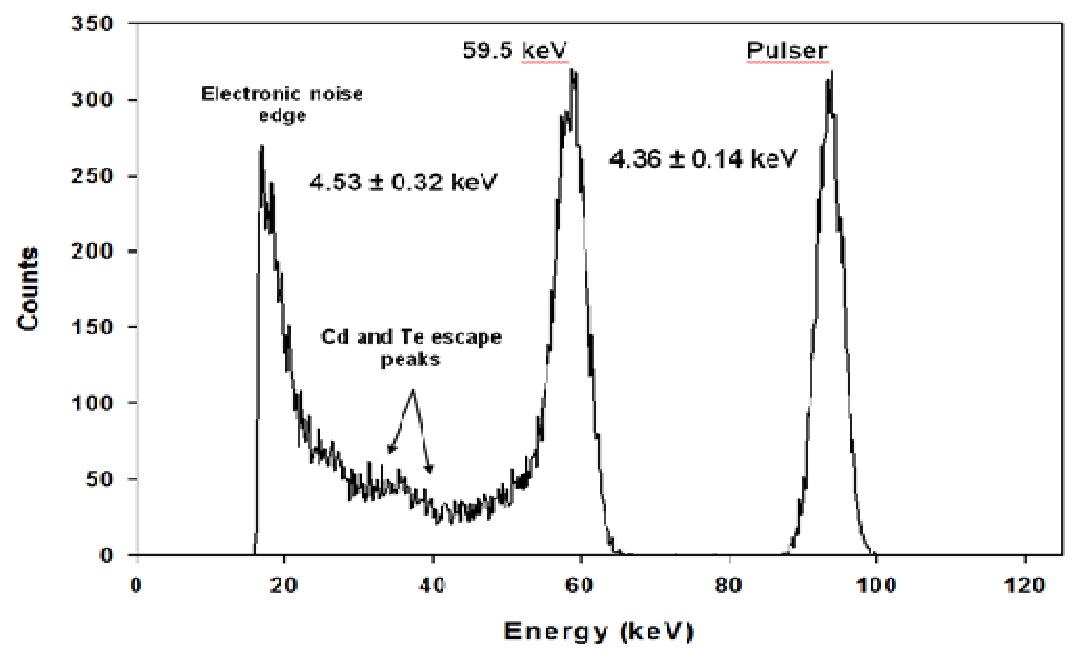

Figure 2: ${ }^{241} \mathrm{Am}(59.5 \mathrm{keV})$ spectrum at $-100 \mathrm{~V}(-10,-20,-30) \mathrm{V}$ 


\section{Results and Discussion}

\subsection{Spectroscopic Performance}

Figure 2 shows an example of a $59.5 \mathrm{keV}$ spectrum at $-100 \mathrm{~V}$ bulk field and $(-10,-20,-30)$ $\mathrm{V}$ on the 1st, 2nd and 3rd ring respectively. The leakage current on the anode at this voltage combination is equal to $1.22 \mathrm{nA}$. The $59.5 \mathrm{keV}$ is clearly visible as well as two smaller features between 30 and $36 \mathrm{keV}$ that are believed to be due to $\mathrm{Cd}$ and Te escape peaks. The pulser width at this combination is nearly equal to the $59.5 \mathrm{keV}$ photo-peak width.

As the bulk and lateral fields are increased, the leakage current on the anode is increased and subsequently the energy resolution of photo-peak is degraded. Figure 3 shows the pulser widths as a function of anode leakage currents for all voltage combinations studied.

The measured pulser width is a product of the intrinsic noise in the electronic read-out chain and the detector leakage current. The preamplifier noise in isolation was measured by generating a pulse through the test input, with the detector input left unconnected; in this arrangement the FWHM was $1.63 \pm 0.01 \mathrm{keV}$. On connecting the preamplifier to the detector system with the detector at $0 \mathrm{~V}$, the pulser FWHM was found to be equal to $3.7 \pm 0.01 \mathrm{keV}$. The broadening of the FWHM despite the lack of a leakage current is due to the additional capacitance of the connecting circuit and cables.

\begin{tabular}{|c|c|c|c|}
\hline $\begin{array}{c}\text { Bulk field } \\
\text { bias on cathode }(\mathrm{V})\end{array}$ & $\begin{array}{c}\text { Lateral field } \\
\text { Bias on rings(1,2 and } \\
3)(\mathrm{V})\end{array}$ & $\begin{array}{c}\text { Leakage current on the } \\
\text { anode }(\mathrm{nA})\end{array}$ & Pulser width (keV) \\
\hline \multirow{2}{*}{-100} & $(-20,-40,-60) \mathrm{V}$ & -1.25 & $4.31 \pm 0.14$ \\
\cline { 2 - 4 } & $(-40,-80,-120) \mathrm{V}$ & -1.98 & $4.59 \pm 0.14$ \\
\cline { 2 - 4 } & $(-80,-160,-240) \mathrm{V}$ & -3.89 & $5.65 \pm 0.14$ \\
\hline-200 & $(-20,-40,-60) \mathrm{V}$ & -3.49 & $5.21 \pm 0.14$ \\
\hline
\end{tabular}

Table 2: Leakage currents and pulser widths for a selection of different voltage combinations.

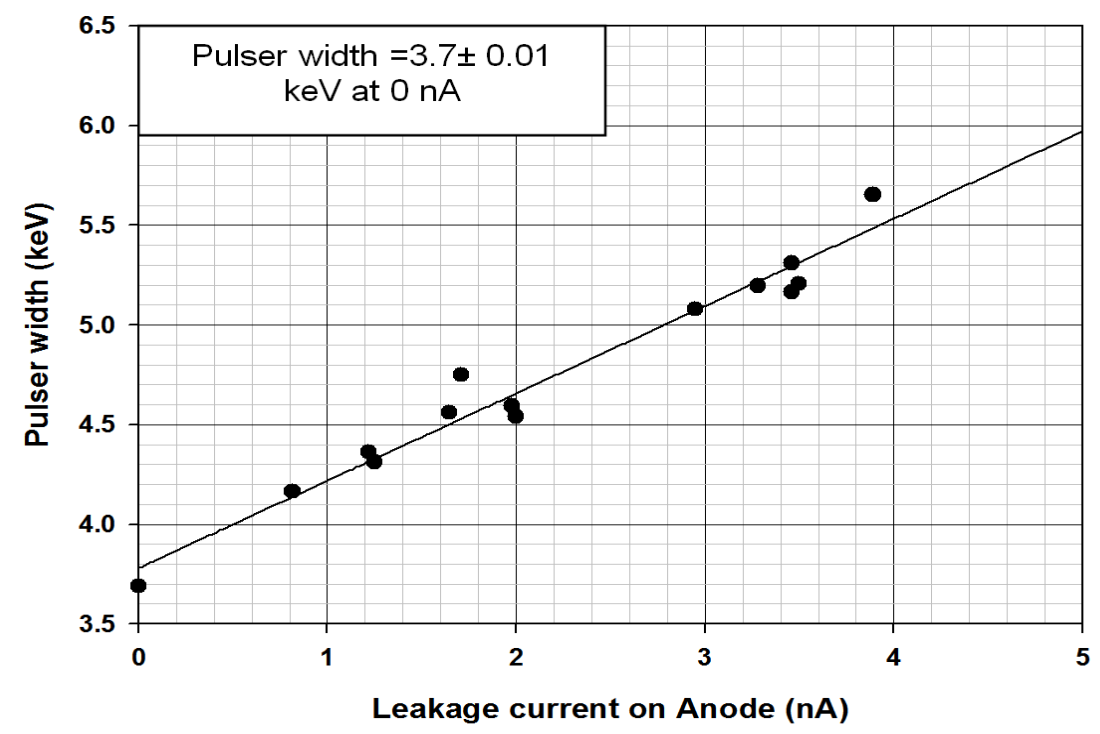

Figure 3: Pulser widths as a function of leakage currents on the anode for all voltage combinations. 
When biasing the detector, the leakage currents on the anode were found to increase with increasing bulk field strength for a fixed lateral field. Increasing the lateral field strength at fixed bulk field also increased the anode leakage current; as indicated in Table 2. It was found that by fixing the $1^{\text {st }}$ ring voltage to $-80 \mathrm{~V}$ and increasing the bias voltages of the $2^{\text {nd }}$ and $3^{\text {rd }}$ rings did not increase the anode leakage current. It is thought that in this instance the $1^{\text {st }}$ ring acts as a guard ring.

\subsection{Microbeam Characterization}

Microbeam measurements were used to investigate the spatial variation of the detector efficiency. The efficiency was evaluated from the count rates detected as a function of radial distance from the anode in a series of line scan measurements. The integrated photo-peak count rates were calculated for each scan position, i.e. from the centre of the anode until the position where the peak count rate is at the level of the noise for the 60 second measurement time; this indicates the active area of the detector. The total count rate integrated over the entire energy range was found to have a large contribution from the low energy noise edge at $\sim 18 \mathrm{keV}$. For this reason only counts in the photo-peak were taken into account for all scans.

Figure 4 shows examples of the $\mathrm{X}$-ray spectra measured for three different positions using $-100 \mathrm{~V}$ bulk field and $(-10,-20,-30) \mathrm{V}$ for rings 1,2 and 3 respectively. The $25 \mathrm{keV}$ peak count rate is at maximum at the centre of the anode and starts to decrease as the scan moves outward from the anode as seen in Figures 4 and 5. The same trend is also observed for the $75 \mathrm{keV}$ photo-peak but with reduced count rates.

Measurements made for fixed lateral bias voltages and varying bulk bias showed no significant effect on the active area of the detector. For example, using ring biases of $-20,-40$, and $-60 \mathrm{~V}$, increasing the bulk field from $-100 \mathrm{~V}$ to $-200 \mathrm{~V}$ had no effect in terms of the active area of the detector which was found to be limited to $0.4 \mathrm{~mm}$ from the anode centre which corresponds to the beginning of the 1st gap. This was confirmed by other measurements with varying bulk field, but fixing lateral fields.

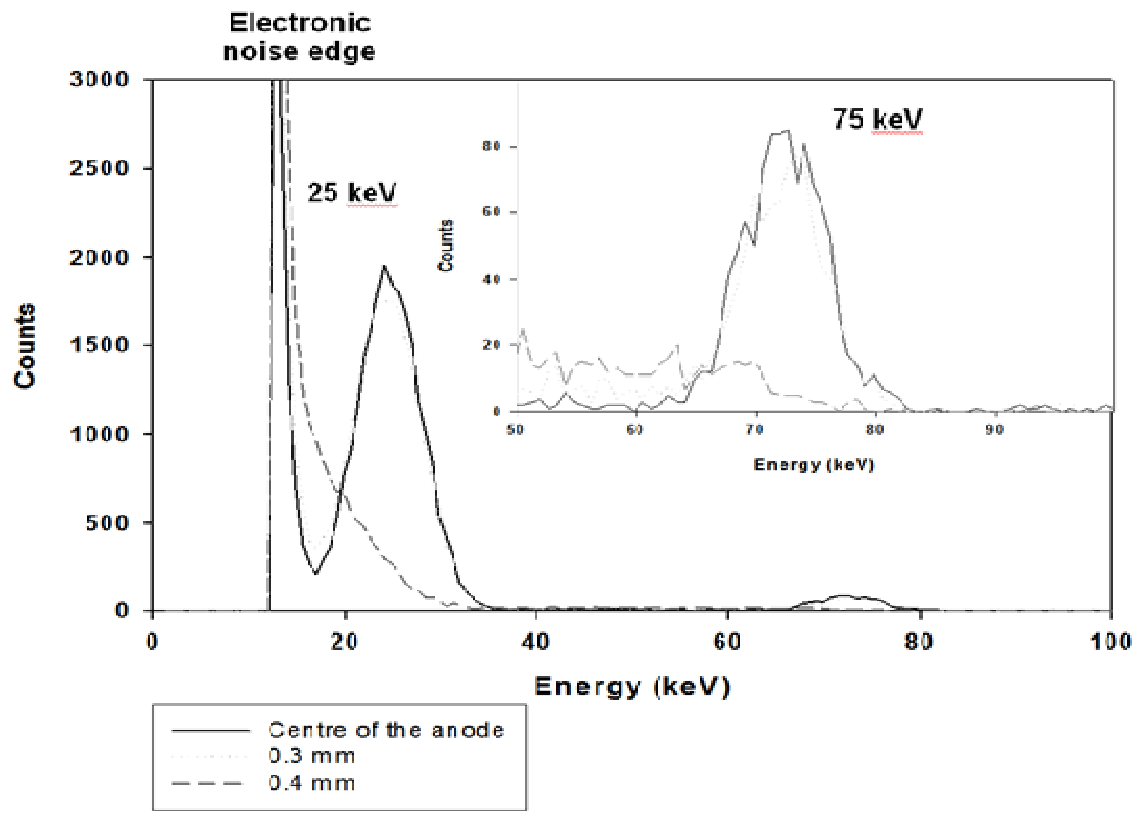

Figure 4 Line scans measurements at -100V $(-10,-20,-30) \mathrm{V}$ 
Figure 5 shows a summary plot for a variety of lateral field combinations at the same bulk field of $-100 \mathrm{~V}$. It is clear from the figure that the choice of biasing scheme is import as the active area of the detector is increased significantly with increased lateral field strength. A maximum active area of $1.9 \mathrm{~mm}$ distance from the anode was achieved at $(-80,-160,-240) \mathrm{V}$ lateral field. When using this bias scheme X-rays are detected in high numbers up to $0.8 \mathrm{~mm}$ from the centre of the anode; beyond this point the number of X-rays detected decreases to $\sim 20$ $\%$ of the maximum between rings 1 and 2 before increasing to $40 \%$ of the maximum when interacting under ring 2 . It was found that the $1^{\text {st }}$ ring bias voltage could not be increased beyond $-80 \mathrm{~V}$ due to an increased contribution of surface leakage currents.

An attempt to further improve the active area of the detector was made by fixing the voltage on the 1 st ring to $-80 \mathrm{~V}$ and increasing the voltages on the $2^{\text {nd }}$ and $3^{\text {rd }}$ rings. Despite the increase in the bias voltages of the outer rings, the active area was found to decrease slightly and this is believed to be due to reduced effective bulk field strength. When the lateral field becomes significantly larger than the bulk field, a drop in count rates was observed due to the high values of 2 nd and 3rd rings which forces electrons away from the anode. These phenomena were also observed for the $75 \mathrm{keV}^{\text {rd }}$ harmonic but with a small reduction in the efficiency compared to the $25 \mathrm{keV}$ photo-peak. Using $(-80,-160,-240) \mathrm{V}$ lateral field, the count rate under the $2^{\text {nd }}$ ring was reduced to $17 \%$ for the $75 \mathrm{keV}$ peak compared to $40 \%$ at $25 \mathrm{keV}$. It appears that the interaction depth plays an important role in terms of detection efficiency. At $75 \mathrm{keV}$ the X-rays interact deeper in the crystal in comparison to $25 \mathrm{keV}$ and this corresponds to a reduced efficiency.

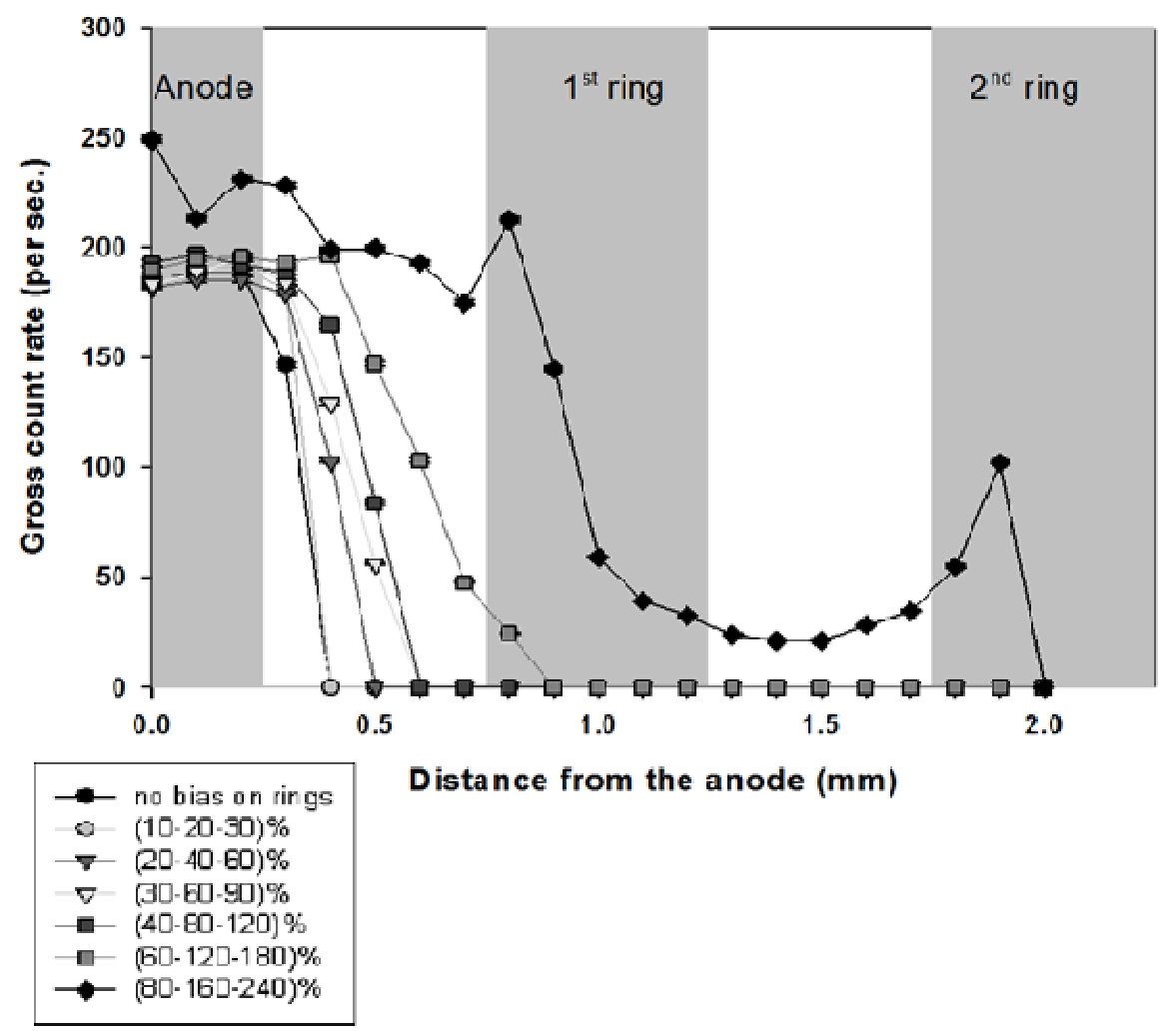

Figure 5 Active area of the detector as a function of lateral fields at $-100 \mathrm{~V}$ bulk field 


\section{Conclusions}

In these measurements the effect of the chosen biasing scheme on the performance of a $\mathrm{CdTe}$ drift ring detector has been investigated. Voltage dependant measurements of the electronic noise showed that the anode leakage current limits the potential detector bias schemes. Under optimised biasing, the FWHM of a $59.5 \mathrm{keV}$ photo-peak was found to be 4.53 $\mathrm{keV}$. Microbeam mapping of the prototype detector demonstrated that the active area extended to the 2nd ring electrode but did not extend beyond this. Line scan measurements show clearly that the active area of the detector is increased significantly by increasing the lateral field. Large surface currents limited the bias of the first ring to $-80 \mathrm{~V}$ while attempts to further increase the active area by increasing the potential on ring 2 and 3 led to a reduction in efficiency due to reduced bulk field strength. For future work, the electronic readout will be optimized to reduce the noise below $2 \mathrm{keV}$. It is expected that the energy resolution will be improved by cooling this detector to lower temperatures to reduce the leakage current. In addition using Schottky contacts of the same geometry could reduce leakage currents, but need to be used at low temperature to reduce the effect of polarization and reach stability [7]. Alternatively a high resistivity material with the same detector geometry, such as CdZnTe, could provide lower leakage currents at room temperature. In terms of counting efficiency, the current geometry can be optimized by decreasing the ring widths and therefore, achieve a large active area.

\section{Acknowledgments}

I gratefully acknowledge King Abdul Aziz University in Saudi Arabia, for funding this project. This work was carried out with the support of Diamond Light Source Ltd. UK.

\section{References}

[1] A. E. Bolotnikov et al., Factors Limiting the Performance of CdZnTe detectors, IEEE Transactions on Nuclear Science, VOl. 52, No.3, June 2005.

[2] Roland den Hartog et al., The CZT Ring-drift detector: A novel concept for hard X-ray detection, in proceeding of Nuclear Science Symposium Conference Record, Vol. 22007 IEEE. DOI:10.1109/NSSMIC.2007.4437284 ISBN: 978-1-4244-0922-8.

[3] M.A.J. Van Pamelen, C. Budtz-Jørgensen, Novel electrode geometry to improve performance of CdZnTe detectors, Nucl. Instrum. Meth., pp, 390-398 (1998).

[4] V. Kondrashov et al., Pulse shape discrimination system for room-temperature semiconductor detectors, in proceedings of 11th Inbt. Workshop on Room Temperature Semiconductor X-and Gamma- Ray Detectors and Associated Electronics, Vol. A458, pp. 580-584, 2001.

[5] E. Gatti and P. Rehak, Semiconductor drift chamber-an application of a novel charge transport scheme, Nucl. Instrum. Meth., 225, 608 (1984).

[6] P. Lechner et al., Silicon drift detectors for high-energy resolution diode type CdTe at room temperature, Nucl. Instrum. Meth., 281-287, (2001).

[7] M. Niraula et al., Stability issues of high-energy resolution diode type CdTe nuclear radiation detectors in a long-term operation, Nucle. Instrum. Meth., 491, 168-175 A (2002).

[8] P. J. Sellin, Detector calibration procedure, May 2001, University of Surrey. 
[9] G. Knoll, Radiation Detection and Measurement, $3^{\text {rd }}$ Johm Wiley \& Sons, Inc. Canada 1998.

[10] K J S Sawhney et al, A Test Beamline on Diamond Light Source, AIP Conference Proceedings Tenth International Conference on Synchrotron Radiation Instrumentation, Australia, 1234, 381 (2010) 